\title{
El perfil de las revistas españolas de comunicación (2007-2008)
}

\author{
David Fernández-Quijada*
}

Resumen: La evolución de los parámetros de publicación científica y de las exigencias de la carrera académica en España ha provocado cambios en las revistas científicas y, entre ellas, las de comunicación. Este artículo caracteriza las revistas nucleares españolas en el ámbito de la comunicación a través de variables como el volumen de artículos publicados, el idioma empleado, la procedencia y las redes de colaboración de sus autores o los patrones de citación en el período 2007-2008. Mediante técnicas bibliométricas y el análisis de redes sociales se obtiene un perfil que muestra las similitudes y las diferencias entre las diferentes revistas y el perfil del conjunto del sistema.

Palabras clave: bibliometría, producción científica, revistas científicas, estudios de comunicación, análisis de redes sociales.

The profile of Spanish journals in the field of communication (2007-2008)

Abstract: The evolution of parameters for scholarly publications and of academic
requirements in Spain has resulted in changes to scholarly journals, among others,
those in the field of communication sciences. This article characterizes the core Spanish
communication journals according to variables such as the number of published
articles, language, author institution and collaboration networks, and citation patterns
during 2007-2008. By applying bibliometric techniques and social network analysis, a
profile showing similarities and differences among the journals is obtained, as well as
a profile of the overall system.

Keywords: Bibliometrics, scholarly production, scholarly journals, communication studies, social network analysis.

\section{Introducción}

El interés entre la comunidad académica española del ámbito de la comunicación por las revistas científicas ha crecido en los últimos años. Muestra de ello es la continua creación de nuevos títulos o los encuentros sobre revistas científicas de comunicación celebrados en la Universitat Autònoma de Barcelona en

* Departament de Comunicació Audiovisual i Publicitat I, Facultat de Ciències de la Comunicació, Universitat Autònoma de Barcelona. Correo-e: david.fernandez@uab.es.

Recibido: 13-10-2009; 2. ${ }^{a}$ versión; 29-3-2010; aceptado: 5-4-2010. 
febrero de 2007 (Perceval, 2007) o en la Universidad Rey Juan Carlos en octubre de 2008. Asimismo, este interés también ha quedado patente en la reflexión sobre la materia, desde posiciones posibilistas (Siguero Guerra, 2008; Fernández Quijada, 2008) o abiertamente críticas (Perceval y Fornieles Alcaraz, 2008). El estudio más significativo sobre la materia hasta la fecha, no obstante, se centró en la valoración que los académicos españoles tenían sobre las revistas del área de periodismo (Giménez Toledo y Alcain Partearroyo, 2006), aunque en realidad la selección temática de títulos las convierte en revistas de comunicación en sentido amplio. Además, en los dos últimos años hasta 3 revistas de este ámbito han entrado en la base de datos de Social Sciences Citation Index: Comunicar, en 2008 y Comunicación y sociedad y Estudios sobre el mensaje periodístico en 2009.

Al hablar sobre la selección que los académicos hacen de las revistas en las que publicar los resultados de su producción científica, el estudio de Giménez Toledo y Alcain Partearroyo (2006: 107) recuerda que "lo que antes era una decisión marcada por las circunstancias, por el tema de investigación o por las preferencias hacia determinadas revistas, se convierte ahora en algo más premeditado, fruto de una planificación, encaminada a una mejor valoración del currículo personal". Es decir, que esta selección también se ha visto dirigida por las exigencias de las diferentes agencias de evaluación de la actividad del profesorado universitario, cuyos criterios han privilegiado las revistas científicas como canal preferente de publicación académica frente a la "cultura de la monografía" (Fernández Quijada, 2008: 6) imperante en algunos ámbitos de las ciencias sociales. Un fenómeno que incluso ha sido bautizado como "efecto ANECA" (Soriano, 2008) en referencia a la agencia evaluadora española, la Agencia Nacional de Evaluación de la Calidad y Acreditación.

En este contexto han surgido numerosos índices bibliométricos y bases de datos bibliográficas como los españoles RESH, MIAR, DICE, Carhus, IN-RECS o el internacional SCIMago Journal \& Country Rank (SJR) que han venido a sumarse a los ya existentes como el latinoamericano Latindex o el clásico Journal Citation Reports (JCR), con diferentes metodologías y grados de desarrollo (Giménez-Toledo y otros, 2007). Los investigadores pueden utilizar estos índices para valorar las revistas y compararlas entre sí, mas su multiplicación provoca dudas sobre cuál es la valoración real de una revista, que, en muchos casos, depende del índice que se consulte.

\section{Objetivos de la investigación}

Esta investigación se ha organizado en torno a tres objetivos. El primero es caracterizar formalmente la producción científica española en el ámbito de la comunicación, que comprende las áreas de conocimiento oficiales en España de Periodismo y de Comunicación Audiovisual y Publicidad, a partir del análisis de sus principales revistas mediante la utilización de indicadores cuantitativos sobre el volumen de producción científica. 
Asimismo, también se plantea como objetivo identificar los principales centros de producción de la investigación publicada, su distribución en función de las revistas y el grado de atracción que ejercen estas revistas para los investigadores nacionales y foráneos.

Finalmente, el tercer objetivo es definir los patrones de citación de las revistas y determinar las más influyentes para sus autores a partir del análisis de las citaciones en sus artículos científicos.

La satisfacción de estos tres objetivos permite perfilar una imagen bastante aproximada de las revistas nucleares españolas de comunicación y establecer sus diferencias y similitudes básicas.

\section{Metodología}

La aproximación bibliométrica a las revistas científicas de comunicación españolas que se emplea en la presente investigación es de naturaleza cuantitativa. Se trata de atribuir un determinado resultado científico en forma de artículo a su autor o, cuando se utilizan unidades agregadas, a su institución de procedencia, tal como se ha procedido en este caso.

El análisis bibliométrico propuesto se centra en una selección representativa de revistas españolas de comunicación, dejando de lado otras vías de publicación científica como el resto de revistas españolas de comunicación, revistas internacionales de comunicación, revistas de otras áreas de conocimiento, monografías, capítulos de libros, comunicaciones de congresos, etc. No se trata, pues, de una medición de producción o productividad científica de las instituciones o de los investigadores del campo sino más bien de una evaluación y caracterización de los patrones de publicación de las principales revistas de comunicación del país. Su aplicabilidad, en consecuencia, se ciñe a la gestión editorial de las revistas y a la revisión de algunos de los parámetros empleados para la evaluación de la actividad investigadora en comunicación dentro del sistema español de ciencia y tecnología.

El tipo de indicadores bibliométricos planteado en este trabajo se ha empleado profusamente en la investigación desde la biblioteconomía y, dado su carácter transversal a todas las ramas científicas, ha llegado también al ámbito de la comunicación, convertido en este caso en objeto de estudio. Una de las primeras investigaciones que tomó las revistas de comunicación como objeto de estudio fue la de Stead (1978), que realizó un análisis de contenido sobre The Journal of Business Communication. En esta misma línea se encuadran trabajos más recientes como los de Potter y Riddle (2007) y Chambers (1998) sobre The Journal of Media Economics. Otros estudios que igualmente se han centrado en analizar publicaciones concretas son los de Freimuth y otros (2006) sobre Journal of Health Communication o los de Dominick (1997), Pardun (2000) y Rice y otros (1996) sobre Journal of Broadcasting E Electronic Media. Ejemplos recientes en el ámbito iberoamericano son el de Chaviano (2007) sobre Signo y pensamiento

Rev. Esp. Doc. Cient., 33, 4, octubre-diciembre, 553-581, 2010. ISSN: 0210-0614. doi:10.3989/redc.2010.4.756 555 
o el de Colle (2009) sobre la Revista latina de comunicación social. Otras investigaciones intentan delimitar subáreas de especialización dentro de la comunicación (Beck y otros, 2004; Lowry y otros, 2007; Miller y Gandy Jr., 1991; Rentz, 2009; Rogers y otros, 2007) o su núcleo (Leydesdorff y Probst, 2009). Existen trabajos similares al que aquí se propone que tratan de dibujar un perfil de publicaciones y, entre otros aspectos, relacionar éstas con las universidades de procedencia de los autores (Bunz, 2005; Kramer y otros, 2007; Lauf, 2005; Masip, 2005) e, incluso, analizar su productividad (Henthorne y otros, 1998; Hickson III y otros, 1999) o su proceso de revisión (Neuman y otros, 2008). En esta línea cabe mencionar también investigaciones previas sobre patrones de citación entre revistas (Feeley, 2008; Poor, 2009).

Con referencia a algunas de estas investigaciones previas se planteó el abordaje más adecuado para el objeto de estudio de este trabajo. Así, para determinar las revistas a analizar se seleccionaron como fuentes las dos bases de datos de revistas españolas que ANECA suele utilizar como referencia al evaluar el ámbito de la comunicación: DICE y Latindex. A éstas se añadió IN-RECS al ser la única que facilita datos sobre el impacto de las mismas mediante análisis de citas.

Se decidió triangular estas bases de datos para obtener un grupo de revistas nucleares de comunicación, aquellas que aparecían en estos 3 índices de referencia con fecha 31 de marzo de 2009. De DICE se vaciaron las áreas de conocimiento de Periodismo y de Comunicación Audiovisual y Publicidad, obteniéndose una lista de 27 revistas. De la sección de Comunicación de IN-RECS se obtuvo la lista de 22 publicaciones que formaban su población. Finalmente, del ámbito de Ciencias de la Comunicación del Catálogo Latindex se obtuvieron 16 revistas editadas en España. Además, se hizo una búsqueda de las revistas aparecidas en la lista de DICE o IN-RECS que faltaban en esta nueva lista. De esta manera emergieron 8 revistas adicionales clasificadas en áreas como Sociología, Bellas Artes, Ciencias de la Información, Lingüística o Cine.

Al cruzar los registros extraídos de las 3 bases de datos mencionadas se obtuvo una lista de 15 revistas que pueden ser consideradas nucleares en el ámbito de la comunicación, aquéllas que aparecen en las 3 bases de datos utilizadas como fuente (anexo I) ${ }^{1}$. Estas 15 revistas fueron seleccionadas como fuente para elaborar la presente investigación a partir de la explotación de sus registros de artículos de los años 2007 y 2008. Estos años fueron elegidos por ser los más recientes con datos completos disponibles. La elección de dos años de muestra perseguía limitar los posibles sesgos que se pudieran producir por fenómenos puntuales como el incumplimiento de la periodicidad de las publicaciones. Siempre que fue posible se emplearon las versiones electrónicas de los artículos y se consultó la versión en papel sólo en aquellos casos de embargo editorial o de archivos inaccesibles. Al recoger la muestra se comprobó que el último número

${ }^{1}$ En el caso de Questiones publicitarias, que cambió de formato papel a electrónico en 2007 con el consiguiente cambio de ISSN, se consideró que existe una continuidad y que la revista es la misma.

556 Rev. Esp. Doc. Cient., 33, 4, octubre-diciembre, 553-581, 2010. ISSN: 0210-0614. doi:10.3989/redc.2010.4.756 
publicado de Mediatika correspondía al año 2005, de manera que la población final de revistas analizadas fue de 14.

Una vez hecha la selección de la muestra, se procedió a la creación de una base de datos elaborada ad hoc. Las variables de esta base de datos se determinaron en función de los objetivos fijados inicialmente. Así, para cumplir con el primer objetivo de caracterización de las revistas se vaciaron campos como el año de publicación, el volumen de la revista, los autores y el título y el idioma de cada artículo. Para el segundo objetivo se vaciaron los datos sobre las instituciones y los países de procedencia de los autores. Finalmente, para cumplir con el tercer objetivo de determinar las revistas más influyentes para los autores españoles, se vaciaron las citaciones a revistas científicas de cada uno de los artículos, vaciando también el nombre de sus autores, su título y el año y el número en que se publicaron. La idea que subyace bajo este planteamiento es que las citaciones permiten análisis relacionales a partir de la concepción de estas citas como una forma de reconocimiento de los autores de los artículos hacia sus pares (Leydesdorff, 1998). La herramienta utilizada para aplicar este análisis de redes sociales (ARS) fue Ucinet 6 (Borgatti y otros, 2002) y NetDraw como programa para la representación gráfica de los sociogramas que muestran las relaciones. Todo ello a partir de una base de datos que una vez completada generó un total de 2.501 registros.

\section{Resultados}

La explotación de la base de datos se realizó a partir de los objetivos de investigación planteados. En primer término, pues, se valoraron diversos aspectos cuantitativos de la producción científica para caracterizarla. Con posterioridad se analizaron las autorías. Finalmente, se establecieron las principales influencias en forma de citaciones.

\subsection{Caracterización}

Las revistas seleccionadas para el análisis aparecen con periodicidad variable. Así, 6 de ellas publican un único número al año (Ámbitos, CIC, Estudios sobre el mensaje periodístico, Historia y comunicación social, I/C y Questiones publicitarias), además de la Revista latina de comunicación social, que formalmente publica un único número pero que se adapta a su naturaleza digital y publica los textos según los acepta. Otras 5 revistas tienen periodicidad semestral (Anàlisi², Comunicación y sociedad, Comunicar, Trípodos y Zer), mientras otra pasó de anual a semestral en el período de muestra (Doxa). Queda otra de aparición

${ }^{2}$ A pesar de mantener nominalmente la periodicidad semestral, en el año 2007 apareció un único número.

Rev. Esp. Doc. Cient., 33, 4, octubre-diciembre, 553-581, 2010. ISSN: 0210-0614. doi:10.3989/redc.2010.4.756 557 
cuatrimestral (Área abierta) y Mediatika, de irregularidad declarada y sin ningún ejemplar durante el período analizado.

Las entidades editoras de estas revistas nucleares de comunicación se concentran en dos instituciones: la Universidad Complutense, responsable de 4 revistas, y la Universidad de Sevilla, editora de 3 más. El resto pertenece a la Universitat Autònoma de Barcelona, la Universidad de Navarra, el Grupo Comunicar, la Universidad San Pablo CEU, Eusko Ikaskuntza, la Universidad de La Laguna, la Universitat Ramon Llull y la Universidad del País Vasco/Euskal Herriko Unibertsitatea.

\subsubsection{Volumen de producción científica}

La producción científica publicada en las 14 revistas de la muestra sumó 289 artículos en 2007 y 395 en 2008, un incremento de un 36,68 \% en tan solo un año que totalizó 684 artículos para el período analizado. El reparto de los textos entre las diferentes revistas fue muy desigual, ya que casi una cuarta parte de esos artículos (167) se publicó en una única revista, Comunicar, que fue además la principal responsable de la inflación de artículos detectada al casi triplicar el número de textos de un año para otro (tabla I). Las siguientes revistas se sitúan a gran

\section{TABLA I}

Número de artículos publicados

\begin{tabular}{l|c|c|c|c|c}
\hline \multicolumn{1}{c|}{ Revista } & $\mathbf{2 0 0 7}$ & $\mathbf{2 0 0 8}$ & $\mathbf{2 0 0 7 - 2 0 0 8}$ & $\mathbf{\%}$ & $\Delta$ \\
\hline Comunicar & 47 & 120 & 167 & 24,42 & +73 \\
\hline Trípodos & 37 & 29 & 66 & 9,65 & -8 \\
\hline EsMP & 30 & 35 & 65 & 9,50 & +5 \\
\hline RLCS & 21 & 42 & 63 & 9,21 & +21 \\
\hline Zer & 32 & 31 & 63 & 9,21 & -1 \\
\hline Ámbitos & 26 & 22 & 48 & 7,02 & -4 \\
\hline Anàlisi & 12 & 25 & 37 & 5,41 & +13 \\
\hline Doxa & 11 & 21 & 32 & 4,68 & +10 \\
\hline AA & 15 & 15 & 30 & 4,39 & 0 \\
\hline I/C & 15 & 13 & 28 & 4,09 & -2 \\
\hline CIC & 12 & 13 & 25 & 3,65 & +1 \\
\hline CyS & 12 & 12 & 24 & 3,51 & 0 \\
\hline HyCS & 12 & 12 & 24 & 3,51 & 0 \\
\hline QP & 7 & 5 & 12 & 1,75 & -2 \\
\hline Media & 20,64 & 28,21 & 48,86 & - & $+7,57$ \\
\hline Total & $\mathbf{2 8 9}$ & $\mathbf{3 9 5}$ & $\mathbf{6 8 4}$ & $\mathbf{1 0 0}$ & $+\mathbf{1 0 6}$ \\
\hline
\end{tabular}

Nota: los códigos de cada revista pueden comprobarse en el anexo II. 
distancia, ya que publican 100 artículos menos que Comunicar. De hecho, esta revista es responsable de tres cuartas partes del incremento en el número de artículos publicados, que aumenta en más de 100 en un solo año. También contribuyeron en gran medida a este incremento Revista latina de comunicación social y el número adicional que publicaron Anàlisi y Doxa. En el lado contrario se sitúa Questiones publicitarias, que en ambos años fue la revista que publicó un menor número de textos, un total de 12 , lo que representa menos de un $2 \%$ del total. El número medio de artículos por revista fue de 48,86 para el período, aunque se aprecia un crecimiento de casi 8 puntos entre el primer y el segundo año.

\subsubsection{Idioma}

Respecto al idioma de los artículos, el castellano es utilizado de manera hegemónica: 627 de los 684 artículos de la muestra fueron escritos en este idioma, lo que representa el 91,67\% del total (tabla II). Este porcentaje tan elevado no le impide ganar casi dos puntos de cuota en 2008 respecto a 2007. Además, es el único idioma en 5 de las revistas analizadas. Tras el castellano se sitúa el catalán, idioma utilizado en 36 artículos, un 5,26\% del total. Excepto 3 textos aparecidos en Anàlisi, el resto se publicó en Trípodos, revista en que éste es el idioma predominante: el $50 \%$ de sus artículos se escribió en catalán por un 48,48\% firmado en castellano. El inglés se empleó en 11 textos, repartidos entre Doxa (4), I/C, Zer (2), Ámbitos, Estudios sobre el mensaje periodístico y Trípodos (1). También tuvo presencia el portugués con 8 textos, 5 de ellos en Comunicar, 2 en Revista latina de comunicación social y 1 en $I / C$. Finalmente, en Comunicar también apareció un texto en francés y otro en italiano.

TABLA II

Idioma de los artículos publicados

\begin{tabular}{|c|c|c|c|c|c|c|c|}
\hline \multirow{2}{*}{ Idioma } & \multicolumn{2}{|c|}{2007} & \multicolumn{2}{|c|}{2008} & \multicolumn{3}{|c|}{$2007-2008$} \\
\hline & n..$^{o}$ & $\%$ & n. ${ }^{\circ}$ & $\%$ & n. ${ }^{\circ}$ & $\%$ & $\Delta(\%)$ \\
\hline Castellano & 262 & 90,66 & 365 & 92,41 & 627 & 91,67 & $+1,75$ \\
\hline Catalán & 20 & 6,92 & 16 & 4,05 & 36 & 5,26 & $-2,87$ \\
\hline Inglés & 5 & 1,73 & 6 & 1,52 & 11 & 1,61 & $-0,21$ \\
\hline Portugués & 2 & 0,69 & 6 & 1,52 & 8 & 1,17 & $+0,83$ \\
\hline Francés & - & - & 1 & 0,25 & 1 & 0,15 & $+0,25$ \\
\hline Italiano & - & - & 1 & 0,25 & 1 & 0,15 & $+0,25$ \\
\hline Total & 289 & 100 & 395 & 100 & 684 & 100 & - \\
\hline
\end{tabular}

${ }^{3}$ En el caso de Zer cabría añadir los 7 artículos en inglés aparecidos en Zer english edition, una experiencia sin solución de continuidad que apareció en el n. ${ }^{\circ} 23$. A pesar de compartir ISSN, no se han contabilizado estos textos que, además, llevan su propia paginación. 
Parece, pues, que a pesar de experiencias como Zer english edition o la recién aparecida Infoamérica-ICR (Iberoamerican Communication Review), las revistas españolas de comunicación sólo emplean de forma testimonial el inglés, una estrategia de internacionalización que revistas españolas de otras áreas sí que han puesto en marcha (Bordons y Gómez, 2004).

\subsection{Autoria}

Además del número de artículos se consideró también el número de autores que firmaba esos textos. Esta cifra sumó 928 nombres en los dos años analizados. Sin las duplicaciones de autores que publicaron más de un texto, en estas 14 revistas firmaron 721 investigadores, aumentando de media más de 10 investigadores por revista. El reparto es algo diferente al del número de artículos y se explica al hacer el cálculo del número de autores por artículo (tabla III). Este índice de coautoría varía entre el 1,04 de CIC e Historia y comunicación social y el 1,83 de Questiones publicitarias. La cifra media se sitúa en 1,36 autores por texto, una cifra que se debe considerar teniendo en cuenta por ejemplo que la revista Comunicar limita el número de autores a un máximo de 3.

TABLA III

Número de autores

\begin{tabular}{l|c|c|c|c|c|c}
\hline \multicolumn{1}{c|}{ Revista } & $\mathbf{2 0 0 7}$ & $\mathbf{2 0 0 8}$ & $\mathbf{2 0 0 7 - 2 0 0 8}$ & $\mathbf{\%}$ & $\Delta$ & $\begin{array}{c}\text { Índice de coautoría } \\
\text { (2007-2008) }\end{array}$ \\
\hline Comunicar & 58 & 157 & 215 & 23,17 & +99 & 1,29 \\
\hline EsMP & 44 & 60 & 104 & 11,21 & +16 & 1,60 \\
\hline Zer & 53 & 50 & 103 & 11,10 & -3 & 1,63 \\
\hline RLCS & 30 & 59 & 89 & 9,59 & +29 & 1,41 \\
\hline Trípodos & 40 & 40 & 80 & 8,62 & 0 & 1,21 \\
\hline Ambitos & 40 & 29 & 69 & 7,44 & -11 & 1,44 \\
\hline Anàlisi & 13 & 36 & 49 & 5,28 & +23 & 1,32 \\
\hline Doxa & 20 & 25 & 45 & 4,85 & +5 & 1,41 \\
\hline I/C & 18 & 18 & 36 & 3,88 & 0 & 1,29 \\
\hline CyS & 14 & 19 & 33 & 3,56 & +5 & 1,38 \\
\hline AA & 16 & 16 & 32 & 3,45 & 0 & 1,07 \\
\hline CIC & 12 & 14 & 26 & 2,80 & +2 & 1,04 \\
\hline HyCS & 12 & 13 & 25 & 2,69 & +1 & 1,04 \\
\hline OP & 14 & 8 & 22 & 2,37 & -6 & 1,83 \\
\hline Media & 27,43 & 38,86 & 66,29 & - & $+11,43$ & 1,36 \\
\hline Total & $\mathbf{3 8 4}$ & $\mathbf{5 4 4}$ & $\mathbf{9 2 8}$ & $\mathbf{1 0 0}$ & $+\mathbf{1 6 0}$ & - \\
\hline
\end{tabular}


Como se puede observar, la cifra está muy próxima a 1, que de hecho es la manera en la que se firman 525 de los 684 artículos de la muestra, un 76,75\% del total. Esta proporción bascula desde el 58,33\% de los artículos de Questiones publicitarias hasta el $96 \%$ de los de CIC. Una colaboración entre dos autores se da en 109 textos, un 15,94\% del total. Dentro de la muestra, el número máximo de autores que firma un artículo es de 6, situación que se da en 4 ocasiones repartidas entre Zer (2), Ámbitos (1) y Estudios sobre el mensaje periodístico (1).

Excepto en el caso de Ámbitos, Questiones publicitarias y Zer, que retroceden, y Área abierta, I/C y Trípodos, que mantienen los mismos datos, la tendencia en la mayoría de revistas es al incremento en el número de autores. En algunos casos, este incremento es coherente con el aumento en el número de originales publicados, como bien ejemplifican Comunicar, Revista latina de comunicación social o Anàlisi, que muestra el impacto de publicar un número más en 2008.

De hecho, la colaboración científica en la autoría de los artículos pone de manifiesto la necesidad de establecer un método de fraccionamiento de la producción científica, es decir, la manera de repartir los resultados entre los coautores. Ante esta disyuntiva son posibles diferentes opciones, como la asignación exclusiva al primer autor, fracción igual para cada autor, más al primero que al resto, fracción decreciente por orden de firma, etc. (Maltrás Barba, 2003). En esta investigación se ha empleado el criterio de la asignación completa, es decir, a cada coautor se le ha atribuido el resultado completo, lo que provoca una lógica redundancia estadística aunque evita el oscurecimiento de las colaboraciones científicas que provoca el resto de criterios posibles.

\subsubsection{Procedencia de los autores}

El análisis de la autoría permite conocer el nombre de los autores más productivos entre las revistas de la muestra y su distribución por centros de procedencia. Al agrupar a estos autores es posible detectar cuáles son las instituciones con mayor producción científica en las revistas nucleares españolas de comunicación. En la mayoría de casos se trata de universidades — de las que procede el 90,95\% de los autores-, aunque también se encuentran otros centros de investigación (el Consejo Superior de Investigaciones Científicas o el Instituto de Estudios de la Democracia), centros de educación preuniversitaria - concentrados en Comunicar-, empresas del sector (Telefónica, Televisión Española, las televisiones autonómicas de Cataluña o las Islas Baleares, El País, La Vanguardia, etc.) u otras instituciones públicas (Consejo Audiovisual de Andalucía, Fundación Audiovisual de Andalucía, Generalitat de Catalunya o el Ministerio Federal de Educación, Ciencia, y Cultura de Austria).

El recuento de la producción científica de estas instituciones sitúa en primer lugar a la Universidad Complutense de Madrid, tanto en 2007 como en 2008, con aproximadamente un $10 \%$ de los autores de las 14 revistas analizadas (tabla IV). Para el conjunto del período, a continuación se sitúan la Universitat Autònoma de Barcelona (8,08\%), la Universidad del País Vasco/Euskal Herriko Unibertsita-

Rev. Esp. Doc. Cient., 33, 4, octubre-diciembre, 553-581, 2010. ISSN: 0210-0614. doi:10.3989/redc.2010.4.756 561 
tea $(6,57 \%)$, la Universidad de Sevilla $(6,57 \%)$ y la Universidad Rey Juan Carlos $(3,34 \%)$, todas ellas públicas. La primera universidad privada que aparece es la Universitat Ramon Llull, en sexta posición, con un 3,13\%. La primera universidad extranjera es la peruana Universidad de Piura, en decimonoveno lugar con un 0,97\%. Estos dos últimos casos, no obstante, ponen de manifiesto dos sesgos detectados en el estudio. En el primero, la concentración de artículos en una única revista y, concretamente, la revista que edita la propia universidad. Así, 22

TABLA IV

Reparto de autorías por instituciones

\begin{tabular}{|c|c|c|c|c|c|c|c|c|c|c|c|}
\hline \multicolumn{4}{|c|}{2007} & \multicolumn{4}{|c|}{2008} & \multicolumn{4}{|c|}{ 2007-2008 } \\
\hline Pos. & Instit. & n. ${ }^{\mathbf{~}}$ & $\%$ & Pos. & Instit. & n. ${ }^{\circ}$ & $\%$ & Pos. & Instit. & n. $^{\circ}$ & $\%$ \\
\hline 1 & UCM & 40 & 10,42 & 1 & UCM & 52 & 9,56 & 1 & UCM & 92 & 9,91 \\
\hline 2 & UAB & 34 & 8,85 & 2 & UAB & 41 & 7,54 & 2 & UAB & 75 & 8,08 \\
\hline 3 & US & 33 & 8,59 & 3 & EHU & 33 & 6,07 & 3 & EHU & 61 & 6,57 \\
\hline 4 & EHU & 28 & 7,29 & 4 & US & 28 & 5,15 & & US & 61 & 6,57 \\
\hline 5 & URL & 21 & 5,47 & 5 & URJC & 25 & 4,60 & 5 & URJC & 31 & 3,34 \\
\hline \multirow[t]{2}{*}{6} & UC3M & 12 & 3,13 & 6 & UMA & 17 & 3,13 & 6 & URL & 29 & 3,13 \\
\hline & UNAV & 12 & 3,13 & 7 & UC3M & 16 & 2,94 & 7 & UC3M & 28 & 3,02 \\
\hline 8 & UPF & 9 & 2,34 & 8 & UHU & 14 & 2,57 & 8 & UNAV & 26 & 2,80 \\
\hline 9 & UMA & 8 & 2,08 & & UNAV & 14 & 2,57 & 9 & UMA & 25 & 2,69 \\
\hline \multirow[t]{2}{*}{10} & USC & 7 & 1,82 & 10 & ULL & 13 & 2,39 & 10 & UPF & 19 & 2,05 \\
\hline & UDEP-PER & 7 & 1,82 & 11 & UPF & 10 & 1,84 & 11 & ULL & 18 & 1,94 \\
\hline \multirow[t]{3}{*}{12} & URJC & 6 & 1,56 & 12 & USAL & 9 & 1,65 & 12 & UHU & 14 & 1,51 \\
\hline & USPCEU & 6 & 1,56 & & USJ & 9 & 1,65 & & USAL & 14 & 1,51 \\
\hline & UDG-MEX & 6 & 1,56 & 14 & UA & 8 & 1,47 & & USC & 14 & 1,51 \\
\hline \multirow[t]{4}{*}{15} & ULL & 5 & 1,30 & & URL & 8 & 1,47 & 15 & USJ & 13 & 1,40 \\
\hline & UPSA & 5 & 1,30 & 16 & UM & 7 & 1,29 & 16 & UA & 12 & 1,29 \\
\hline & USAL & 5 & 1,30 & & USC & 7 & 1,29 & 17 & USPCEU & 9 & 0,97 \\
\hline & UVIGO & 5 & 1,30 & 18 & UOC & 6 & 1,10 & & UVIGO & 9 & 0,97 \\
\hline \multirow[t]{7}{*}{19} & UA & 4 & 1,04 & 19 & $\mathrm{UCH}$ & 5 & 0,92 & & UDEP-PER & 9 & 0,97 \\
\hline & USJ & 4 & 1,04 & & UGR & 5 & 0,92 & 20 & UJI & 8 & 0,86 \\
\hline & UV & 4 & 1,04 & & UJI & 5 & 0,92 & & UM & 8 & 0,86 \\
\hline & UDEC-CHI & 4 & 1,04 & & UVIC & 5 & 0,92 & & UOC & 8 & 0,86 \\
\hline & Otras & 109 & 28,39 & & Otras & 189 & 34,74 & & UPSA & 8 & 0,86 \\
\hline & nd & 10 & 2,60 & & nd & 18 & 3,31 & & Otras & 309 & 33,30 \\
\hline & Total & 384 & 100 & & Total & 544 & 100 & & Total & 928 & 100 \\
\hline
\end{tabular}

Nota: Los códigos de cada institución pueden comprobarse en el anexo III. 
de las 29 autorías atribuidas a investigadores de la Universitat Ramon Llull proceden de Trípodos, un 75,86\% del total. En el segundo caso, 7 de las 9 autorías de la Universidad de Piura corresponden a una única investigadora, que con su productividad consigue arrastrar al conjunto de su institución. A ello cabría añadir el hecho de que la tendencia de los académicos del área a publicar en las revistas de su propia universidad ha impulsado el resultado tanto de la Universidad Complutense de Madrid como de la Universidad de Sevilla, que aparece sobredimensionado.

Además de la mera asignación y cuantificación de autorías se procedió también a realizar un análisis relacional entre las revistas analizadas y la universidad de procedencia de los autores. Para focalizar el análisis en los principales centros de producción se eliminaron las instituciones cuyos autores habían publicado solamente en una o dos revistas. Así, se distribuyeron relacionalmente las revistas analizadas y un total de 39 instituciones cuyos autores habían publicado a lo largo de 2007 y 2008 en al menos 3 de las 14 revistas de la muestra (figura 1).

\section{FIGURA 1}

\section{Instituciones de procedencia de los autores}

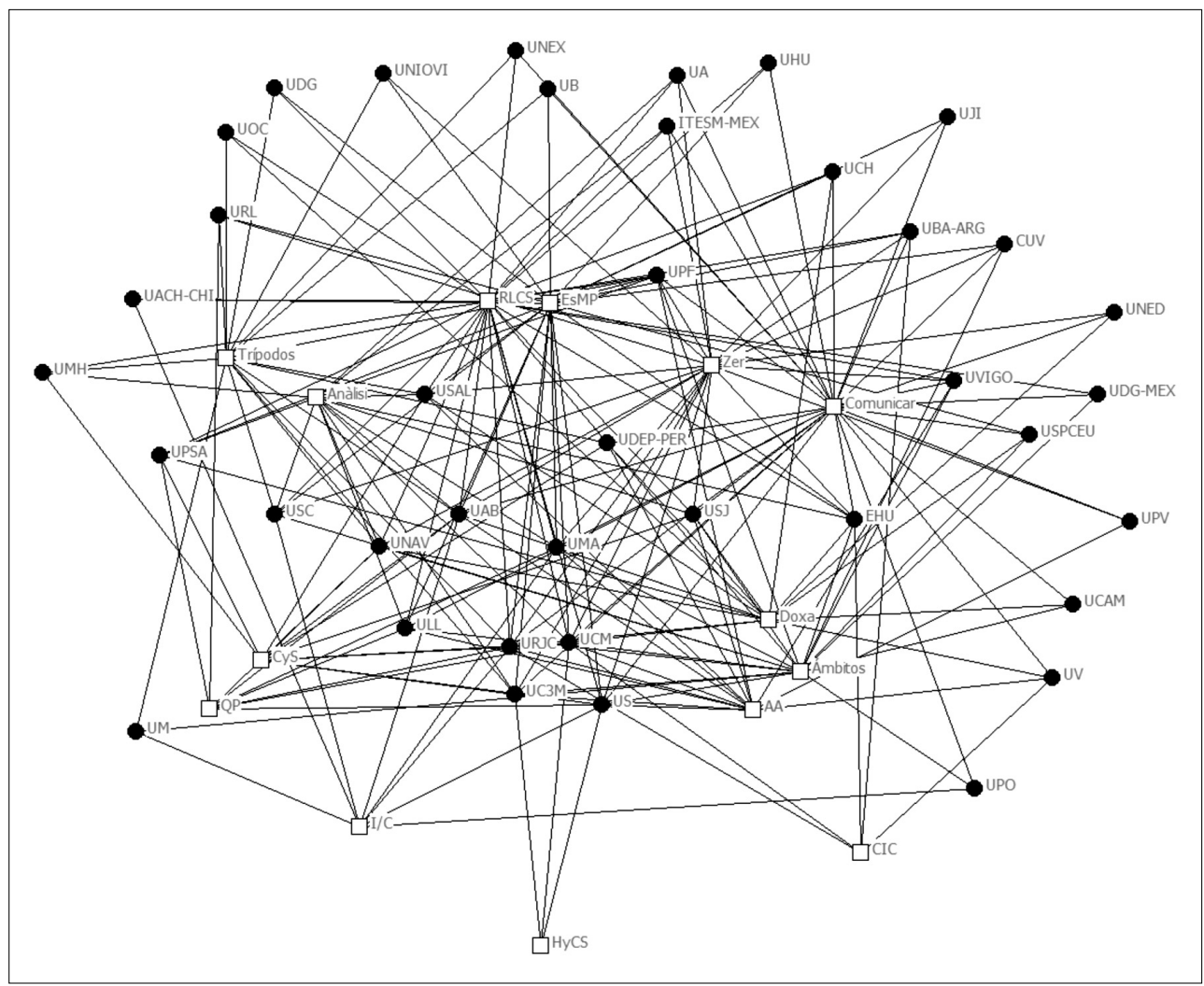

Rev. Esp. Doc. Cient., 33, 4, octubre-diciembre, 553-581, 2010. ISSN: 0210-0614. doi:10.3989/redc.2010.4.756 563 
Sólo 5 de estas instituciones eran extranjeras: las mexicanas ITESM y Universidad de Guadalajara, la peruana Universidad de Piura, la Universidad de Buenos Aires, Argentina y la Universidad Austral de Chile. El resultado permite observar los patrones de relación entre universidades y revistas, así como el número de relaciones y la centralidad tanto de revistas como de universidades. En general, las revistas con mayor número de textos ocupan las posiciones centrales. Aquí el volumen parece un factor clave, al revés que en el caso de las universidades, cuya centralidad depende más de la variedad de publicaciones y de dirigirlas a revistas centrales: esa es la razón que explica la centralidad de la Universidad de Piura o de la Universitat Pompeu Fabra aún sin contar con un gran número de textos. Entre las revistas, la distribución arquetípica de procedencias la ofrecían títulos como Zer, Revista latina de comunicación social o Estudios sobre el mensaje periodistico.

Un dato a destacar es el grado de internacionalización de los autores de las diferentes revistas. La revista más internacionalizada en cuanto a autoría es CIC, en la que un $30,77 \%$ de los autores es foráneo. Le siguen muy de cerca Comunicar (30,70\%) e $I / C(27,78 \%)$. Por el contrario, la menos internacionalizada es Questiones publicitarias, en la que todos sus autores proceden de universidades españolas, a la que siguen Trípodos (2,50\%) y Anàlisi (6,12\%). La procedencia de estos autores extranjeros se sitúa mayoritariamente en Latinoamérica, que en su conjunto representa el 12,28\% de todas las autorías. Dentro de este ámbito destacan México (2,91\%), Argentina (2,37\%), Chile (1,51\%), Brasil (1,51\%), Colombia (1,19\%) y Perú (1,08\%). De la Unión Europea procede el 4,74\% de los autores, principalmente de Italia $(0,86 \%)$, Francia $(0,75 \%)$, Portugal $(0,75 \%)$ y Reino Unido $(0,65 \%)$. Otros países cuyas cifras merecen un apunte son Estados Unidos $(0,97 \%)$ y Canadá $(0,32 \%)$. La representación relacional de esta aportación internacional a las revistas españolas dibuja el importante papel jugado por Comunicar - lógico dado su alto volumen de textos-, aunque los patrones de relación los marcan los países que han publicado en mayor número de revistas centrales, como Perú, Brasil, Chile, Reino Unido o Argentina y las revistas que mayor variedad de orígenes tienen y en las que más publican los autores de los países centrales, como Ámbitos o Revista latina de comunicación social (figura 2).

\subsubsection{Redes de colaboración}

Casi una cuarta parte de todos los artículos analizados, un total de 159, estaban firmados por más de un autor. La mayoría de coautorías pertenecían a la misma institución siendo la colaboración interinstitucional un fenómeno poco frecuente que alcanzaba la cota de prácticamente inexistente en colaboraciones entre más de dos instituciones. De hecho, al trazar los patrones de relaciones a partir de esta colaboración institucional se observa una red muy dispersa y poco densa (figura 3). La colaboración entre la Universidad de Navarra y la Univer-

564 Rev. Esp. Doc. Cient., 33, 4, octubre-diciembre, 553-581, 2010. ISSN: 0210-0614. doi:10.3989/redc.2010.4.756 
FIGURA 2

Países de procedencia de los autores extranjeros

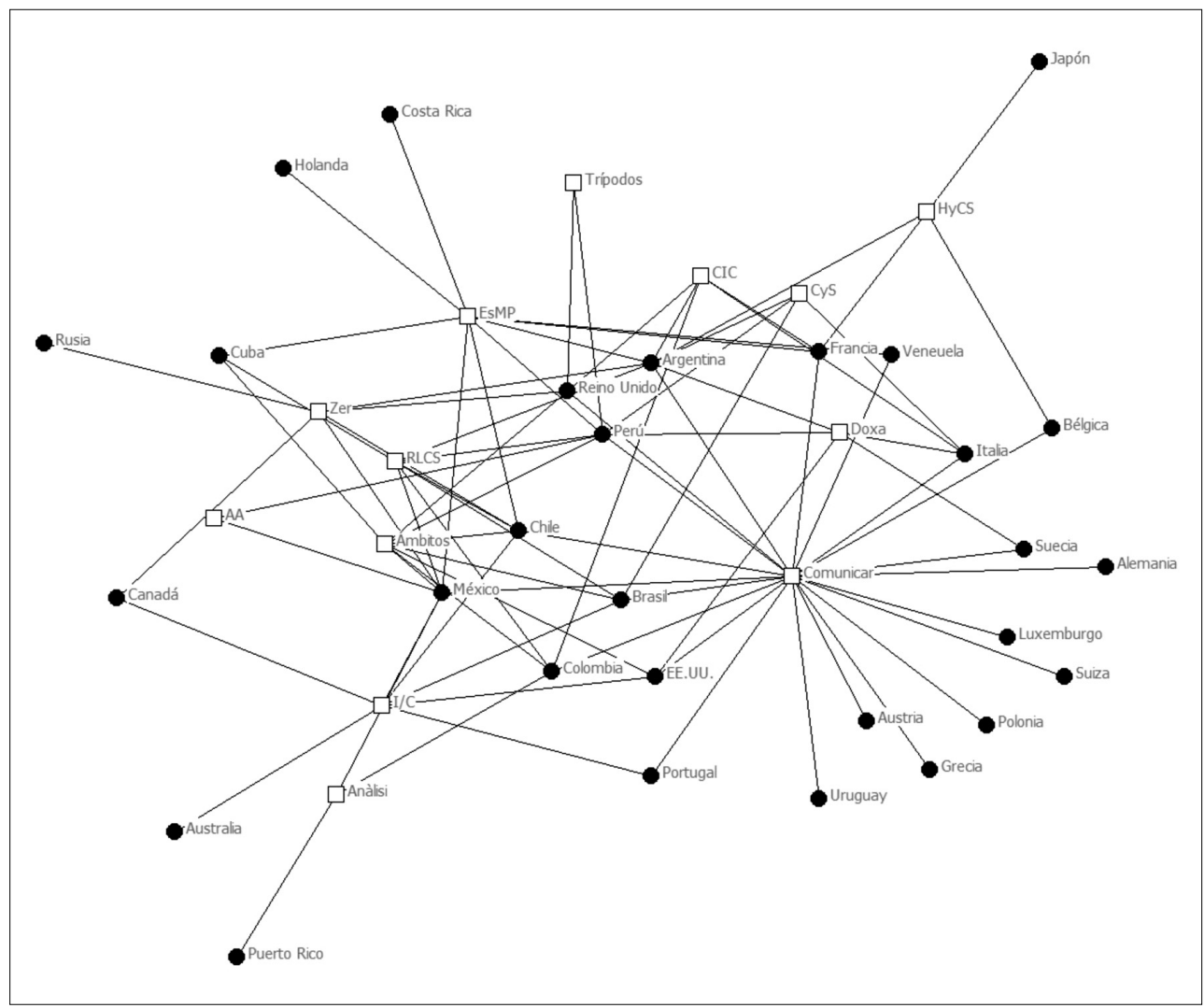

sidad Miguel Hernández, por un lado, y entre la Universitat Oberta de Catalunya y la Universidad San Jorge, por otro, se da en 3 ocasiones mientras que el resto de los ejemplos son únicos. Las universidades en que se da más colaboración con otras instituciones son la Universidad del País Vasco, con 6 colaboraciones distintas, la Universidad de Navarra y la Universidad de Huelva, con 4, y la Universidad Complutense de Madrid, la Universidad de Murcia, la Universitat Pompeu Fabra, la Universidad San Pablo CEU, la Universidad Austral argentina y la peruana Universidad de San Martín de Porres, con 3 cada una. El mayor número de colaboraciones internacionales, por otro lado, se da en los casos de la Universidad del País Vasco con instituciones mexicanas, argentinas y peruanas y de la Universidad de Navarra con dos centros colombianos y uno brasileño. 
FIGURA 3

Colaboración interinstitucional de los autores

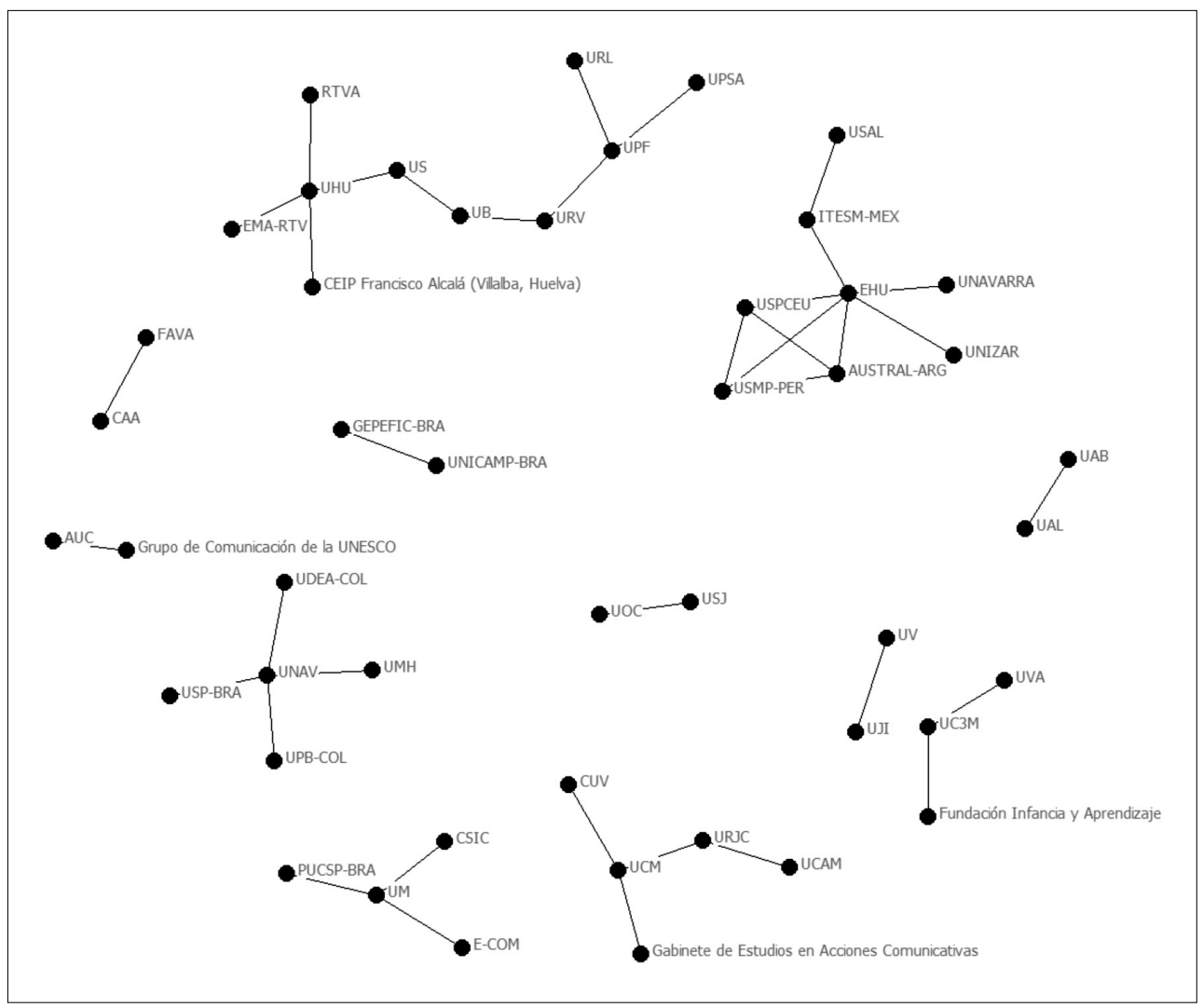

\subsection{Patrones de citación}

Las citas que plasman los autores en sus artículos académicos reflejan la influencia que reciben de estos trabajos. La agrupación de estas citas en función de la revista en la que se publicaron originalmente los artículos citados permite conocer el grado de influencia que ejerce la revista citada sobre los autores que publican en la citante, en este caso las revistas de la muestra.

Para ello es fundamental conocer un primer parámetro: el volumen de citas generado por las revistas de la muestra (tabla V). Entre los años 2007 y 2008 esta cifra prácticamente se duplica, pasando de 760 a 1.491 . Mientras que algunas revistas mantienen estable el número de citas generadas (Zer o $\mathrm{I} / \mathrm{C}$ ) y unas pocas generan menos citas (Ámbitos la mitad e Historia y comunicación social apenas genera), la mayoría aumenta el número de citas. Entre éstas destacan aumentos que doblan (Anàlisi, Área abierta, CIC o Comunicar), cuadriplican (Trípodos), 


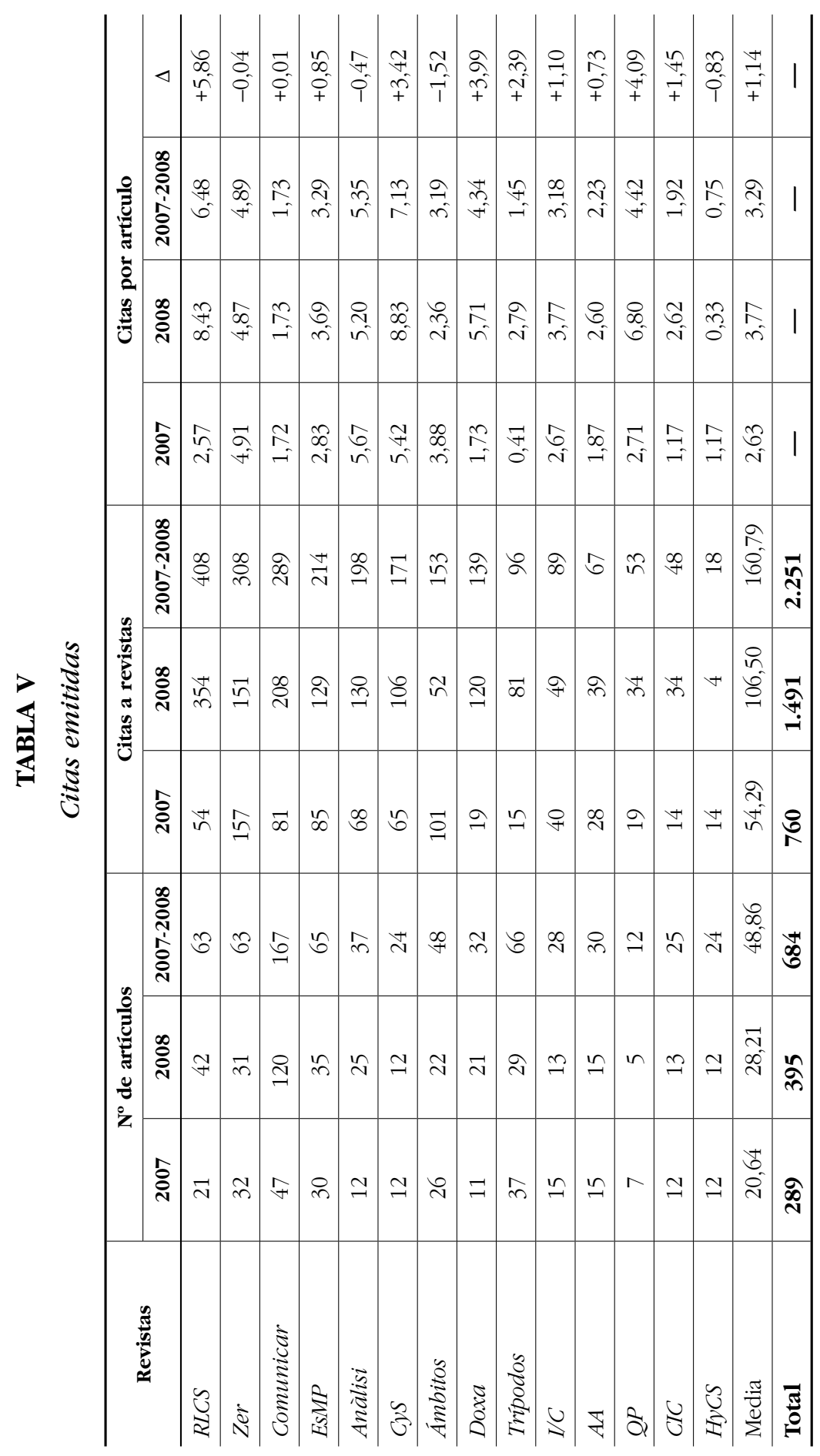


sextuplican (Doxa) o septuplican (Revista latina de comunicación social) el número de citas del año anterior, sin que en algunos casos exista correspondencia con el aumento en el número de textos publicados.

El cruce de estos dos últimos datos permite conocer el número de citas generado de media en cada artículo. Esta lista la encabeza Comunicación y sociedad con 7,13 citas a revistas científicas por cada artículo publicado, seguida por Revista latina de comunicación social $(6,48)$. En el lado contrario de esta lista se sitúan Historia y comunicación social $(0,75)$, Trípodos $(1,45)$ y Comunicar (1,73). En algunos casos, además, este incremento en el número de citas por artículo es poco menos que espectacular, como Revista latina de comunicación social, que pasa de 2,57 a 8,43 citas por artículo. También es el caso de Questiones publicitarias, aunque el bajo número de artículos publicados desvirtúa este dato, que puede deberse a artículos concretos que tienen un gran peso sobre el total.

Si se analiza el destino de estas citaciones se obtiene un listado de las revistas que más influyen en los investigadores que publican su trabajo en las revistas analizadas (tabla VI). Así, la revista más citada es la Revista latina de comunicación social, que suma 133 citas, casi un 6\% del total, con un enorme salto entre 2007 (7 citas) y 2008 (126 citas). La segunda más citada es Comunicar (93), justo por delante de Zer (87). Cuarta se sitúa la primera revista extranjera, Journal of Communication (60), por delante de Telos (57), Estudios sobre el mensaje periodístico (45) y Anàlisi, Comunicación y sociedad y European Journal of Communication (30), que junto a Ámbitos (26) ocupan las 10 primeras posiciones. Entre las 20 primeras también aparecen otras revistas de la muestra como Trípodos (21), CIC (19) o Área abierta (18), además de las primeras revistas latinoamericanas, Sala de prensa (21) y Chasqui (20).

Se detectan incrementos espectaculares de citación como las 119 citas que añade la Revista latina de comunicación social, que tiene su explicación en su elevada tasa de autocitas, que supone 89 de estas nuevas citaciones. También Comunicar $(+51)$ y Zer $(+27)$ consiguen incrementos importantes y, entre las extranjeras, Journal of Communication suma más de 20 referencias adicionales.

Al centrar el foco en las autocitas se observa que esta cifra se sitúa de media en 17,14 por revista y una clara tendencia alcista, al triplicarse entre 2007 (4) y $2008(13,14)$ (tabla VII). Si se calcula el porcentaje de autocitas dividiendo éstas entre el número total de citas recibidas aparecen algunos cifras muy elevadas, por encima del 50\%, como Área abierta (77,78\%), Revista latina de comunicación social (68,42\%) y Comunicar (64,52\%). Especialmente significativo es el caso de Revista latina de comunicación social, que entre 2007 y 2008 pasa de 1 autocita a 90, es decir, de representar el 14,29\% de todos sus impactos a suponer el 71,43\%. Esta revista y Comunicar (60 autocitas) arrastran al conjunto de la muestra al crecimiento en el número de autocitas, ya que entre las 12 restantes las autocitas sólo aumentan en 9, 8 de ellas responsabilidad de Área abierta, la otra revista con un alto porcentaje de autocitas. Estudios sobre el mensaje perio-

568 Rev. Esp. Doc. Cient., 33, 4, octubre-diciembre, 553-581, 2010. ISSN: 0210-0614. doi:10.3989/redc.2010.4.756 
TABLA VI

Revistas más citadas

\begin{tabular}{|c|c|c|c|c|c|c|}
\hline Pos. & Revista & 2007 & 2008 & $2007-2008$ & $\%$ & $\Delta$ \\
\hline 1 & RLCS & 7 & 126 & 133 & 5,91 & +119 \\
\hline 2 & Comunicar & 21 & 72 & 93 & 4,13 & +51 \\
\hline 3 & Zer & 30 & 57 & 87 & 3,86 & +27 \\
\hline 4 & JoC & 18 & 42 & 60 & 2,67 & +24 \\
\hline 5 & Telos & 21 & 36 & 57 & 2,53 & +15 \\
\hline 6 & $E s M P$ & 17 & 28 & 45 & 2,00 & +11 \\
\hline \multirow[t]{3}{*}{7} & Anàlisi & 9 & 21 & 30 & 1,33 & +12 \\
\hline & CyS & 14 & 16 & 30 & 1,33 & +2 \\
\hline & $E J C$ & 13 & 17 & 30 & 1,33 & +4 \\
\hline 10 & Ámbitos & 9 & 17 & 26 & 1,16 & +8 \\
\hline 11 & SexRoles & 4 & 21 & 25 & 1,11 & +17 \\
\hline 12 & $J B E E M$ & 3 & 20 & 23 & 1,02 & +17 \\
\hline \multirow[t]{2}{*}{13} & $S d P$ & 9 & 12 & 21 & 0,93 & +3 \\
\hline & Trípodos & 5 & 16 & 21 & 0,93 & +11 \\
\hline \multirow[t]{2}{*}{15} & Chasqui & 7 & 13 & 20 & 0,89 & +6 \\
\hline & $C R$ & 9 & 11 & 20 & 0,89 & +2 \\
\hline 17 & CIC & 13 & 6 & 19 & 0,84 & -7 \\
\hline 18 & $A A$ & 5 & 13 & 18 & 0,80 & +8 \\
\hline 19 & Gazette & 3 & 13 & 16 & 0,71 & +10 \\
\hline \multirow[t]{5}{*}{20} & $J S$ & 4 & 11 & 15 & 0,67 & +7 \\
\hline & MCES & 8 & 7 & 15 & 0,67 & -1 \\
\hline & $Q d C A C$ & 6 & 9 & 15 & 0,67 & +3 \\
\hline & Otras & 525 & 907 & 1.432 & 63,62 & +382 \\
\hline & Total & 760 & 1.491 & 2.251 & 100 & +731 \\
\hline
\end{tabular}

dístico también se sitúa cerca del 50\% de autocitas pero, al contrario que Revista latina de comunicación social, su tendencia es descendente. De media, las autocitas representan el $46,33 \%$ de todas las citas recibidas por las principales revistas españolas de comunicación, aumentando seis puntos y medio entre 2007 y 2008. La parte inferior de este listado la ocupan $I / C$, la única publicación que no recibe citas ni autocitas, Trípodos, Ámbitos, Zer y Comunicación y sociedad, justo en la barrera del $20 \%$. 
TABLA VII

Autocitas

\begin{tabular}{l|c|c|c|c|c|c|c|c|c|c}
\hline \multirow{2}{*}{ Revista } & \multicolumn{3}{|c|}{$\mathbf{2 0 0 7}$} & \multicolumn{3}{c|}{$\mathbf{2 0 0 8}$} & \multicolumn{4}{c}{$\mathbf{2 0 0 7 - 2 0 0 8}$} \\
\cline { 2 - 12 } & Citas & Autoc. & $\mathbf{\%}$ & Citas & Autoc. & $\mathbf{\%}$ & Citas & Autoc. & \% & $\Delta$ \\
\hline AA & 5 & 3 & 60 & 13 & 11 & 84,62 & 18 & 14 & 77,78 & +8 \\
\hline RLCS & 7 & 1 & 14,29 & 126 & 90 & 71,43 & 133 & 91 & 68,42 & +89 \\
\hline Comunicar & 21 & 15 & 71,43 & 72 & 45 & 62,50 & 93 & 60 & 64,52 & +30 \\
\hline EsMP & 17 & 14 & 82,35 & 28 & 8 & 28,57 & 45 & 22 & 48,89 & -6 \\
\hline Doxa & 4 & 1 & 25 & 4 & 2 & 50 & 8 & 3 & 37,50 & +1 \\
\hline Anàlisi & 9 & 2 & 22,22 & 21 & 8 & 38,10 & 30 & 10 & 33,33 & +6 \\
\hline CIC & 13 & 4 & 30,77 & 6 & 2 & 33,33 & 19 & 6 & 31,58 & -2 \\
\hline HyCS & 1 & 0 & - & 3 & 1 & 33,33 & 4 & 1 & 25 & +1 \\
\hline QP & 0 & 0 & - & 4 & 1 & 25 & 4 & 1 & 25 & +1 \\
\hline CyS & 14 & 4 & 28,57 & 16 & 2 & 12,50 & 30 & 6 & 20 & -2 \\
\hline Zer & 30 & 7 & 23,33 & 57 & 10 & 17,54 & 87 & 17 & 19,54 & +3 \\
\hline Ambitos & 9 & 4 & 44,44 & 17 & 1 & 5,88 & 26 & 5 & 19,23 & -3 \\
\hline Trípodos & 5 & 1 & 20 & 16 & 3 & 18,75 & 21 & 4 & 19,05 & +2 \\
\hline I/C & 0 & 0 & - & 0 & 0 & - & 0 & 0 & - & 0 \\
\hline Media & 9,64 & 4 & 41,48 & 27,36 & 13,14 & 48,04 & 37 & 17,14 & 46,33 & $+9,14$ \\
\hline Total & $\mathbf{1 3 5}$ & $\mathbf{5 6}$ & - & $\mathbf{3 8 3}$ & $\mathbf{1 8 4}$ & - & $\mathbf{5 1 8}$ & $\mathbf{2 4 0}$ & - & $+\mathbf{1 2 8}$ \\
\hline
\end{tabular}

Las tres primeras revistas en porcentaje de autocitas son también las tres primeras en porcentaje de autorreferencias, es decir, autocitas de entre el total de citaciones a revistas que genera esa publicación (tabla VIII). Así, Revista latina de comunicación social tiene un 22,30\% de autorreferencias, lo que significa que 22 de cada 100 citas a revistas generadas en sus artículos son autocitas. Este dato es aún más significativo si se ve la evolución entre 2007 y 2008. De hecho, en 2007 este porcentaje era el más bajo de todas las revistas analizadas, un escaso 1,85\%, correspondiente a una única autocita, pero en 2008 crece hasta más allá del 25\%. También Área abierta (20,90\%) y Comunicar (20,76\%) sitúan su porcentaje de autorreferencias más allá del 20\%, con un crecimiento importante en el primer caso, que concentra 10 de sus 11 autocitas de ese año en su último número, el 21. Estas cifras son más del doble de la media de la muestra, fijada en el 10,66\%, en gran medida por el alza que introducen estas 3 revistas, que juntas generan el $68,75 \%$ de todas las autocitas. Cerca de la media de autorreferencias se sitúan CIC (12,50\%) y Estudios sobre el mensaje periodístico (10,28\%), 
aunque con una tendencia marcadamente descendente hacia las cifras de la mayoría de revistas, que tienen como referencia más cercana el 5\%.

\section{TABLA VIII}

Autorreferencias

\begin{tabular}{|c|c|c|c|c|c|c|c|c|c|}
\hline \multirow{2}{*}{ Revista } & \multicolumn{3}{|c|}{2007} & \multicolumn{3}{|c|}{2008} & \multicolumn{3}{|c|}{$2007-2008$} \\
\hline & Citas & Autoc. & $\%$ & Citas & Autoc. & $\%$ & Citas & Autoc. & $\%$ \\
\hline RLCS & 54 & 1 & 1,85 & 354 & 90 & 25,42 & 408 & 91 & 22,30 \\
\hline$A A$ & 28 & 3 & 10,71 & 39 & 11 & 28,21 & 67 & 14 & 20,90 \\
\hline Comunicar & 81 & 15 & 18,52 & 208 & 45 & 21,63 & 289 & 60 & 20,76 \\
\hline CIC & 14 & 4 & 28,57 & 34 & 2 & 5,88 & 48 & 6 & 12,50 \\
\hline EsMP & 85 & 14 & 16,47 & 129 & 8 & 6,20 & 214 & 22 & 10,28 \\
\hline HyCS & 14 & 0 & - & 4 & 1 & 25 & 18 & 1 & 5,56 \\
\hline Zer & 157 & 7 & 4,46 & 151 & 10 & 6,62 & 308 & 17 & 5,52 \\
\hline Anàlisi & 68 & 2 & 2,94 & 130 & 8 & 6,15 & 198 & 10 & 5,05 \\
\hline Tripodos & 15 & 1 & 6,67 & 81 & 3 & 3,70 & 96 & 4 & 4,17 \\
\hline CyS & 65 & 4 & 6,15 & 106 & 2 & 1,89 & 171 & 6 & 3,51 \\
\hline Ámbitos & 101 & 4 & 3,96 & 52 & 1 & 1,92 & 153 & 5 & 3,27 \\
\hline Doxa & 19 & 1 & 5,26 & 120 & 2 & 1,67 & 139 & 3 & 2,16 \\
\hline$Q P$ & 19 & 0 & - & 34 & 1 & 2,94 & 53 & 1 & 1,89 \\
\hline$I / C$ & 40 & 0 & - & 49 & 0 & - & 89 & 0 & - \\
\hline Media & 54,29 & 4 & 7,37 & 106,50 & 13,14 & 12,34 & 160,79 & 17,14 & 10,66 \\
\hline Total & 760 & 56 & - & 1.491 & 184 & - & 2.251 & 240 & - \\
\hline
\end{tabular}

Para realizar el análisis relacional de las revistas objeto de citación se escogieron las que hubieran recibido más de 10 citas a lo largo del período analizado - un total de 38 títulos distintos - y a ellas se sumaron las revistas de la muestra que no alcanzaban esa cifra -5 títulos-. El resultado de la aplicación de un análisis de redes sociales a partir de las citaciones ofrece un núcleo de revistas centrales que coincide con el listado de las más citantes, aunque en este caso emiten a la vez que reciben citas, con la excepción de Doxa (figura 4). Conforme se avanza hacia el exterior de la red van apareciendo otros títulos en función del número de citaciones y del número de revistas diferentes desde la que reciben estas citaciones, como Telos o Journal of Communication. 


\section{FIGURA 4}

Citaciones de revistas españolas de comunicación

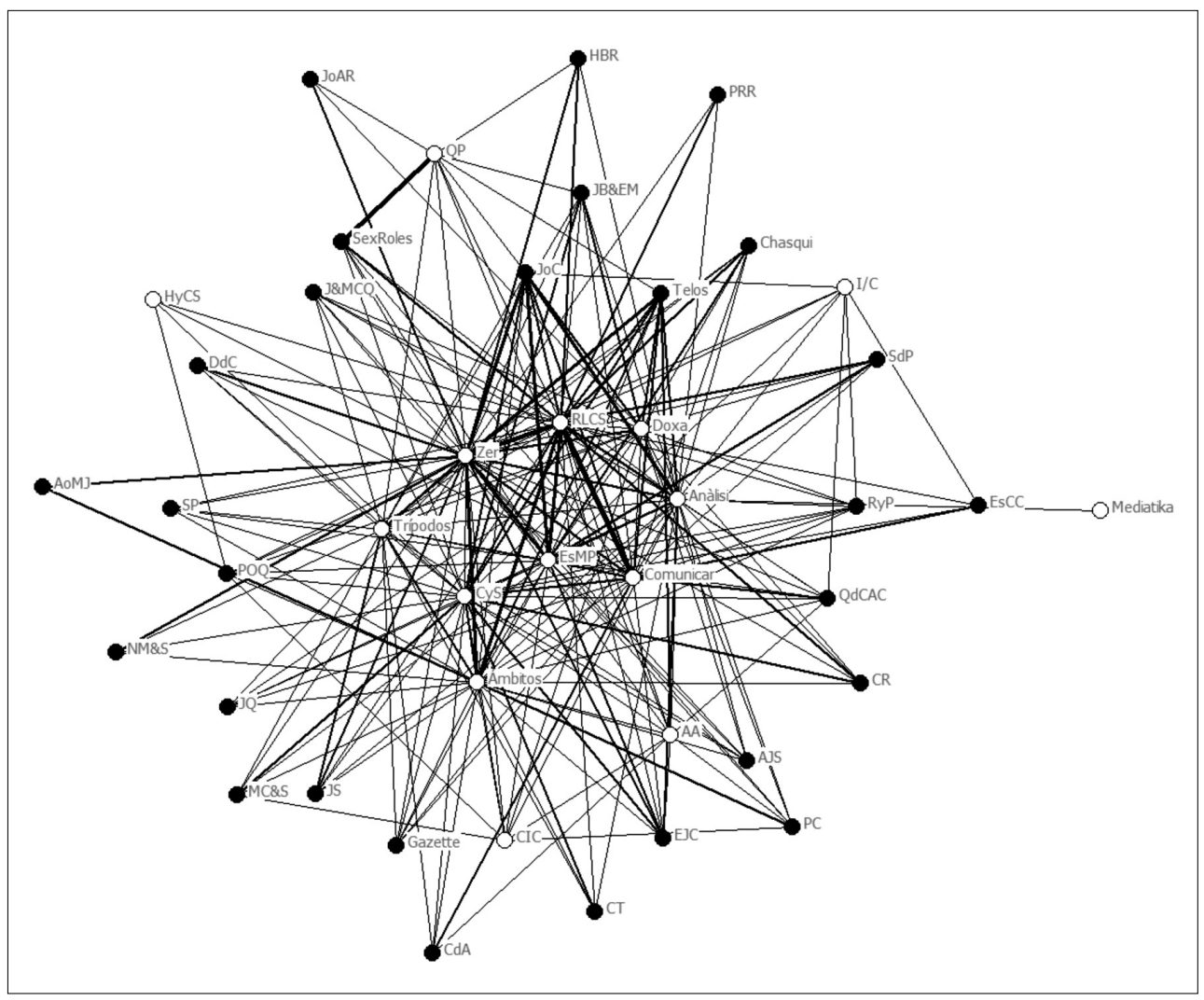

\section{Conclusiones}

El perfil de las revistas españolas de comunicación analizadas en este trabajo ofrece algunos elementos de aparente optimismo, como el destacado incremento del número de originales publicados en 2008 respecto del año anterior, acompañado del consiguiente aumento de autorías. Sin embargo, el análisis de estas autorías es un elemento en el que ya aparecen los primeros claroscuros. Por una parte, las universidades de mayor tradición, como la Universidad Complutense de Madrid, la Universitat Autònoma de Barcelona, la Universidad del País Vasco o la Universidad de Navarra, mantienen un peso importante en términos cuantitativos aunque ello no impide el acceso a las principales publicaciones de autores de universidades de creación más reciente e incluso de autores procedentes de otras áreas de conocimiento. No obstante, pervive aún el modelo de autoría individualizada frente a los trabajos realizados por equipos de investigación, que 
continúan siendo minoritarios en el conjunto de las revistas analizadas. La autoría conjunta es todavía más difícil a nivel interinstitucional, en el que se observa escaso dinamismo a pesar de la amplitud de la muestra utilizada en este trabajo.

La preeminencia del castellano como lengua de publicación en las revistas analizadas se sitúa por encima del 90\%, lo que confirma su buena salud en este ámbito. Sin duda, las revistas españolas orientan su perfil internacional al espacio académico y cultural iberoamericano, del que proceden la mayoría de autores foráneos que publican en sus páginas. A pesar de que muchas de las revistas analizadas tienen una política idiomática abierta que permite, por ejemplo, la publicación en inglés, este idioma apenas es utilizado. La publicación en inglés ofrecería una mayor proyección internacional en el ámbito anglosajón pero también mucha mayor competencia por la atención del potencial lector. Las conexiones con otros entornos internacionales, por tanto, aparecen como bastante débiles.

Un último aspecto que se ha analizado en esta investigación ha sido el de las citaciones y, por extensión, la influencia que tienen las revistas. En cuanto a la generación de citaciones, cada vez se cita más pero aparecen algunos elementos que llaman la atención como crecimientos en las tasas de autocitación que seguramente tengan más que ver con estrategias editoriales en pos de un mayor índice de impacto que con un aumento real de la influencia de estas revistas. Este alto nivel de autocitas tiene su reflejo en la lista de revistas citadas, en la que también se observan llamativos incrementos entre 2007 y 2008. En conjunto, un porcentaje de autocitas cercano al 50\% para el conjunto de revistas de comunicación dibuja un escenario de impacto sustentado artificialmente por la acción de las revistas en que esta práctica es más frecuente, un dato refrendado por el volumen de autorreferencias detectado. El caso de Área abierta, no obstante, plantea algunas dudas por el bajo número de citas de la muestra, que la hace más sensible a sobredimensionar las autocitas, y porque no es una revista fuente en IN-RECS, de manera que sus citas y autocitas no tienen impacto en este índice, el más usado habitualmente.

Otro factor a destacar es la presencia predominante de las revistas españolas entre las más citadas; así, 8 de las 10 primeras son españolas, dejando apenas espacio para dos revistas internacionales de referencia, Journal of Communication y European Journal of Communication. Igualmente sobresale la influencia que mantienen algunas revistas latinoamericanas por delante de revistas españolas o de otras anglosajonas de gran renombre. Ello vendría a demostrar que el ámbito de influencia en el espacio académico iberoamericano de comunicación es bidireccional y que se da cierto aislamiento respecto a la investigación en otros idiomas.

Entre las revistas más citadas y, por tanto, reconocidas por las autores de revistas españolas, aparecen algunas como Telos y Quaderns del CAC que no formaban parte de la muestra a pesar de que aparentemente tienen mayor impacto que otras que sí están indizadas en los índices de referencia. En el caso de Telos, además, esta centralidad es confirmada por el estudio de Giménez Toledo y Alcain Partearroyo (2006), en el que era la revista más relevante para los académicos del área de periodismo. Esta ausencia debería motivar la reflexión y el replanteamiento tanto de las políticas de difusión de estas revistas como de algunos de los criterios

Rev. Esp. Doc. Cient., 33, 4, octubre-diciembre, 553-581, 2010. ISSN: 0210-0614. doi:10.3989/redc.2010.4.756 573 
utilizados en estos índices bibliométricos ${ }^{4}$. A la vez, plantea la necesidad de incluir factores correctores en la selección de la muestra de revistas para futuros estudios.

El trabajo aquí presentado ofrece resultados de potencial aplicación práctica en la gestión editorial de las revistas españolas de comunicación y apunta algunos elementos de interés que podrían ayudar a mejorar los índices y bases de datos bibliométricos, como una mayor monitorización de patrones de citación anormales que podrían ser indicativos de prácticas editoriales poco éticas (Brumback, 2009; Foo, 2009). Aunque limitada a dos años, la muestra es relativamente amplia y ofrece, por tanto, un perfil bastante detallado de un campo extenso como el de la comunicación. Una continuidad temporal en el trabajo aquí presentado y una ampliación a análisis temáticos a partir de palabras clave permitiría perfilar aún más el terreno de las revistas científicas de comunicación, convertidas en elementos centrales de difusión del conocimiento científico y de progreso profesional en el nuevo entorno de la carrera académica española.

\section{Bibliografia}

Beck, C. S.; Benitez, J. L.; Edwards, A.; Olson, A.; Pai, A., y Torres, M. B. (2004). Enacting "Health Communication": The Field of Health Communication as Constructed Through Publication in Scholarly Journals. Health Communication, vol. 16 (4), 475-492.

Bordons, M., y Gómez, I. (2004). Towards a single language in science? A Spanish view. Serials, vol. 17 (2), 189-195.

Borgatti, S. P.; Everett, M. G., y Freeman, L. C. (2002). Ucinet 6 for Windows: Software for Social Network Analysis, Harvard, MA; Analytic Technologies.

Brumback, R. A. (2009). Impact Factor Wars: Episode V - The Empire Strikes Back. Journal of Child Neurology, vol. 24 (3), 260-262.

Bunz, U. (2005). Publish or Perish: A Limited Author Analysis of ICA and NCA Journals. Journal of Communication, vol. 55 (4), 703-720.

Chambers, T. (1998). Who's on First? Studying the Scholarly Community of Media Economics. The Journal of Media Economics, vol. 11 (1), 1-12.

Chaviano, O. G. (2007). Análisis bibliométrico y de calidad de la revista Signo y Pensamiento (1982-2006). Signo y pensamiento, n. 50, 22-32.

Colle, R. (2009). La temática de Revista Latina de Comunicación Social, 1998-2008. Revista latina de comunicación social, n. 64, 71-85, http://www.revistalatinacs.org/09/ art/07_806_13_revista/Raymond_Colle.html [consulta: 22 de agosto de 2009].

Dominick, J.R. (1997). Standpoint: Citation analysis of the Journal of Broadcasting E Electronic Media: Another perspective. Journal of Broadcasting \& Electronic Media, vol. 41 (3), 427-438.

Feeley, T. H. (2008). A Bibliometric Analysis of Communication Journals from 2002 to 2005. Human Communication Research, vol. 34 (3), 505-520.

4 «En la actualización de 2009 de IN-RECS, tras la realización de esta investigación, Telos ha entrado a formar parte de este índice, de manera que a partir de ese momento ya cumplía los criterios muestrales exigidos en esta investigación".

574 Rev. Esp. Doc. Cient., 33, 4, octubre-diciembre, 553-581, 2010. ISSN: 0210-0614. doi:10.3989/redc.2010.4.756 
Fernández Quijada, D. (2008). Revistas científicas e indices de impacto. A propósito de "Hacer saber". Área Abierta, n. 20, 1-10, http://www.ucm.es/BUCM/revistas/inf/15788393/ articulos/ARAB0808230001D.PDF [consulta: 4 de mayo de 2009].

Foo, J. Y. A. (2009). Impact of Excessive Journal Self-Citations: A Case Study on the Folia Phoniatrica et Logopaedica Journal. Science and Engineering Ethics, Online First.

Freimuth, V. S.; Massett, H. A., y Meltzer, W. (2006). A Descriptive Analysis of 10 Years of Research Published in the Journal of Health Communication. Journal of Health Communication, vol. 11 (1), 11-20.

Giménez Toledo, E., y Alcain Partearroyo, M. D. (2006). Estudio de las revistas españolas de periodismo. Comunicación y sociedad, vol. XIX (2), 107-131.

Giménez-Toledo, E.; Román-Román, A., y Alcain-Partearroyo, M. D. (2007). From experimentation to coordination in the evaluation of Spanish scientific journals in the humanities and social sciences. Research Evaluation, vol. 16 (2), 137-148.

Henthorne, T. L.; Latour, M. S., y Loraas, T. (1998). Publication Productivity in the Three Leading U.S. Advertising Journals: 1989 through 1996. Journal of Advertising, vol. XXVII (2), 53-63.

Hickson III, M.; Stacks, D. W., y Bodon, J. (1999). The Status of Research Productivity in Communication: 1915-1995. Communication Monographs, vol. 66 (2), 178-197.

Kramer, M. W.; Hess, J. A., y Reid, L. D. (2007). Trends in Communication Scholarship: An Analysis of Four Representative NCA and ICA Journals over the Last 70 Years. The Review of Communication, vol. 7 (3), 229-240.

Lauf, E. (2005). National Diversity of Major International Journals in the Field of Communication. Journal of Communication, vol. 55 (1), 139-151.

Leydesdorff, L. (1998). Theories of citation? Scientometrics, vol. 43 (1), 5-25.

Leydesdorff, L., y Probst, C. (2009). The Delineation of an Interdisciplinary Speciality in Terms of a Journal Set: The Case of Communication Studies. Journal of the American Society for Information Science and Technology, vol. 60 (8), 1709-1718.

Lowry, P. B.; Humpherys, S. L.; Malwitz, J., y Nix, J. A. (2007). Scientometric Study of the Perceived Quality of Business and Technical Communication Journals. IEEE Transactions on Professional Communication, vol. 50 (4), 352-378.

Maltrás Barba, B. (2003). Los indicadores bibliométricos. Fundamentos y aplicación al análisis de la ciencia. Gijón; Trea.

Masip, P. (2005). European Research in Communication during the years 1994-2004: a Bibliometric Approach. First European Communication Conference, Amsterdam, Holanda: European Communication Research and Education Association [CD-ROM].

Miller, K. M., y Gandy Jr., O. H. (1991). Paradigmatic Drift: A Bibliographic Review of the Spread of Economic Analysis in the Literature of Communication. Journalism Quarterly, vol. 68 (1), 663-671.

Neuman, W. R.; Davidson, R.; Joo, S.-H.; Park, Y. J., y Williams, A. E. (2008). The Seven Deadly Sins of Communication Research. Journal of Communication, vol. 58 (2), 220237.

Pardun, C. J. (2000). An Analysis of Qualitative Research in the Journal of Broadcasting E Electronic Media, 1978-1998. Journal of Broadcasting E Electronic Media, vol. 44 (3), 529-534.

Rev. Esp. Doc. Cient., 33, 4, octubre-diciembre, 553-581, 2010. ISSN: 0210-0614. doi:10.3989/redc.2010.4.756 575 
Perceval, J. M. (2007). I Trobada de Revistes i Publicacions Científiques sobre Comunicació. Anàlisi, n. 35, 273-275.

Perceval, J. M., y Fornieles Alcaraz, J. (2008). Confucio contra Sócrates: la perversa relación entre la investigación y la acreditación. Anàlisi, n. 36, 213-224.

Poor, N. D. (2009). Global Citation Patterns of Open Access Communication Studies Journals: Pushing Beyond the Social Science Citation Index. International Journal of Communication, vol. 3, 853-879, http://ijoc.org/ojs/index.php/ijoc/article/view/568/360 [consulta: 26 de junio de 2009].

Potter, W. J., y Riddle, K. (2007). A Content Analysis of the Media Effects Literature. Journalism \& Mass Communication Quarterly, vol. 84 (1), 90-104.

Rentz, K. (2009). The Importance of Niche Journals to New Business-Communication Academics and to All of Us. Journal of Business Communication, vol. 46 (3), 404-411.

Rice, R. E.; Chapin, J.; Pressman, R.; Park, S., y Funkhouser, E. (1996). Special feature: What's in a name? Bibliometric analysis of 40 years of the Journal of Broadcasting $\mathcal{E}$ Electronic Media. Journal of Broadcasting E Electronic Media, vol. 40 (4), 511-539.

Rogers, P. S.; Campbell, N.; Louhiala-Salminen, L.; Rentz, K., y Suchan, J. (2007). The impact of perceptions of journal quality on business and management communication academics. Journal of Business Communication, vol. 44 (4), 403-426.

Siguero Guerra, M. (2008). Hacer saber. Objetivo final del investigador en comunicación. Área abierta, n. 19, 1-8, http://revistas.ucm.es/inf/15788393/articulos/ ARAB0808110001D.PDF [consulta: 4 de mayor de 2009].

Soriano, J. (2008). El efecto ANECA. Actas y memoria final. Congreso internacional fundacional $A E-I C$, p. 1-18, Santiago de Compostela, España: Asociación Española de Investigación de la Comunicación [CD-ROM].

Stead, B. A. (1978). A Content Analysis of The Journal of Business Communication: Toward a Body of Knowledge. The Journal of Business Communication, vol. 15 (2), 45-51.

\section{ANEXOS}

\section{Anexo I. Triangulación y población de revistas de la muestra}

\begin{tabular}{|c|c|c|c|c|c|}
\hline Revistas & Editor & DICE & $\begin{array}{l}\text { Catálogo } \\
\text { Latindex }\end{array}$ & $\begin{array}{l}\text { IN- } \\
\text { RECS }\end{array}$ & Muestra \\
\hline $\begin{array}{l}\text { Ámbitos. Revista internacional de } \\
\text { comunicación }\end{array}$ & U. de Sevilla & $\mathrm{X}$ & $\mathrm{X}$ & $\mathrm{X}$ & $\mathrm{X}$ \\
\hline $\begin{array}{l}\text { Anàlisi: quaderns de comunicació } \\
\quad \text { i cultura }\end{array}$ & $\begin{array}{l}\text { U. Autònoma de Barce- } \\
\text { lona }\end{array}$ & $\mathrm{X}$ & $\mathrm{X}$ & $\mathrm{X}$ & $\mathrm{X}$ \\
\hline $\begin{array}{l}\text { Archivos de la Filmoteca. Revista de } \\
\text { estudios históricos sobre la ima- } \\
\text { gen }\end{array}$ & Generalitat Valenciana & $\mathrm{X}$ & $\mathrm{XX}^{*}$ & - & - \\
\hline Área abierta & $\begin{array}{l}\text { U. Complutense de Ma- } \\
\text { drid }\end{array}$ & $\mathrm{X}$ & $\mathrm{X}$ & $\mathrm{X}$ & $\mathrm{X}$ \\
\hline
\end{tabular}




\begin{tabular}{|c|c|c|c|c|c|}
\hline Revistas & Editor & DICE & $\begin{array}{l}\text { Catálogo } \\
\text { Latindex }\end{array}$ & $\begin{array}{c}\text { IN- } \\
\text { RECS }\end{array}$ & Muestra \\
\hline $\begin{array}{l}\text { CIC. Cuadernos de información y } \\
\text { comunicación }\end{array}$ & $\begin{array}{l}\text { U. Complutense de Ma- } \\
\text { drid }\end{array}$ & $\mathrm{X}$ & $\mathrm{X}$ & $\mathrm{X}$ & $\mathrm{X}$ \\
\hline $\begin{array}{l}\text { Comunicación y estudios universi- } \\
\quad \text { tarios }\end{array}$ & CEU San Pablo & $\mathrm{X}$ & - & 一 & - \\
\hline $\begin{array}{l}\text { Comunicación y bombre: revista } \\
\text { interdisciplinar de ciencias de la } \\
\text { comunicación y bumanidades }\end{array}$ & U. Francisco de Vitoria & - & $\mathrm{X}$ & $\mathrm{X}$ & - \\
\hline Comunicación y sociedad & U. de Navarra & $\mathrm{X}$ & $\mathrm{X}^{* *}$ & $\mathrm{X}$ & $\mathrm{X}$ \\
\hline $\begin{array}{l}\text { Comunicación. Revista internacio- } \\
\text { nal de comunicación audiovi- } \\
\text { sual, publicidad y estudios cul- } \\
\text { turales }\end{array}$ & U. de Sevilla & $\mathrm{X}$ & - & - & - \\
\hline $\begin{array}{l}\text { Comunicar. Revista científica ibe- } \\
\text { roamericana de comunicación y } \\
\text { educación }\end{array}$ & Grupo Comunicar & $\mathrm{X}$ & $\mathrm{X}$ & $\mathrm{X}$ & $\mathrm{X}$ \\
\hline $\begin{array}{l}\text { Coneixement } i \text { societat: revista } \\
\text { d'universitats, recerca } i \text { societat } \\
\text { de la información }\end{array}$ & $\begin{array}{l}\text { Generalitat de Catalun- } \\
\text { ya }\end{array}$ & - & - & $\mathrm{X}$ & - \\
\hline Doxa comunicación & U. San Pablo-CEU & $\mathrm{X}$ & $\mathrm{X}$ & $\mathrm{X}$ & $\mathrm{X}$ \\
\hline $\begin{array}{l}\text { Estudios sobre el mensaje periodis- } \\
\quad \text { tico }\end{array}$ & $\begin{array}{l}\text { U. Complutense de Ma- } \\
\text { drid }\end{array}$ & $\mathrm{X}$ & $\mathrm{X}^{* * * *}$ & $\mathrm{X}$ & $\mathrm{X}$ \\
\hline Etic@ net & U. de Granada & - & - & $\mathrm{X}$ & - \\
\hline Film-bistoria & $\begin{array}{l}\text { Centro de Investigacio- } \\
\text { nes Film-Historia }\end{array}$ & $\mathrm{X}$ & - & - & - \\
\hline $\begin{array}{l}\text { Formats. Revista de comunicación } \\
\text { audiovisual }\end{array}$ & U. Pompeu Fabra & - & - & $\mathrm{X}$ & - \\
\hline Historia y comunicación social & $\begin{array}{l}\text { U. Complutense de Ma- } \\
\text { drid }\end{array}$ & $\mathrm{X}$ & $\mathrm{X}$ & $\mathrm{X}$ & $\mathrm{X}$ \\
\hline I/C. Información y comunicación & U. de Sevilla & $\mathrm{X}$ & $\mathrm{X}$ & $\mathrm{X}$ & $\mathrm{X}$ \\
\hline Icono 14 & $\begin{array}{l}\text { Asoc. Científica de In- } \\
\text { vestigación de las } \\
\text { NNTT de la Educa- } \\
\text { ción }\end{array}$ & - & $\mathrm{X}$ & $\mathrm{X}$ & - \\
\hline $\begin{array}{l}\text { IDP. Revista de Internet, derecho y } \\
\text { politica }\end{array}$ & U. Oberta de Catalunya & - & $\mathrm{X}$ & - & - \\
\hline $\begin{array}{l}\text { Ikusgaiak. Cuadernos de cinemato- } \\
\text { grafia }\end{array}$ & Eusko Ikaskuntza & $\mathrm{X}$ & $X^{*}$ & - & - \\
\hline $\begin{array}{l}\text { Mediatika. Cuadernos de medios de } \\
\text { comunicación }\end{array}$ & Eusko Ikaskuntza & $\mathrm{X}$ & $\mathrm{X}^{* *}$ & $\mathrm{X}$ & $\mathrm{X}$ \\
\hline $\begin{array}{l}\text { Pensar la publicidad. Revista inter- } \\
\text { nacional de investigaciones pu- } \\
\text { blicitarias }\end{array}$ & $\begin{array}{l}\text { U. Complutense de Ma- } \\
\text { drid }\end{array}$ & $\mathrm{X}$ & $\mathrm{X}$ & - & - \\
\hline
\end{tabular}




\begin{tabular}{|c|c|c|c|c|c|}
\hline Revistas & Editor & DICE & $\begin{array}{l}\text { Catálogo } \\
\text { Latindex }\end{array}$ & $\begin{array}{l}\text { IN- } \\
\text { RECS }\end{array}$ & Muestra \\
\hline $\begin{array}{l}\text { Quaderns de filologia. Estudis de } \\
\text { comunicació }\end{array}$ & U. de València & $\mathrm{X}$ & $\mathrm{X}^{* * * * *}$ & 一 & - \\
\hline Questiones publicitarias & U. de Sevilla & $\mathrm{X}$ & 一 & 一 & - \\
\hline $\begin{array}{l}\text { Questiones publicitarias. Revista in- } \\
\text { ternacional de comunicación y } \\
\text { publicidad }\end{array}$ & U. de Sevilla & $\mathrm{X}$ & $\mathrm{X}$ & $\mathrm{X}$ & $\mathrm{X}$ \\
\hline Red Iris & Red.es / RedIris & - & $\mathrm{X}$ & 一 & - \\
\hline $\begin{array}{l}\text { Revista de ciencias de la informa- } \\
\text { ción }\end{array}$ & $\begin{array}{l}\text { U. Complutense de Ma- } \\
\text { drid }\end{array}$ & $\mathrm{X}$ & 一 & - & 一 \\
\hline $\begin{array}{l}\text { Revista latina de comunicación so- } \\
\text { cial }\end{array}$ & U. de La Laguna & $\mathrm{X}$ & $\mathrm{X}$ & $\mathrm{X}$ & $\mathrm{X}$ \\
\hline $\begin{array}{l}\text { Revista universitaria de publicidad } \\
\text { y relaciones públicas }\end{array}$ & $\begin{array}{l}\text { U. Complutense de Ma- } \\
\text { drid }\end{array}$ & $\mathrm{X}$ & 一 & 一 & 一 \\
\hline $\begin{array}{l}\text { Sphera pública: revista de ciencias } \\
\text { sociales y de la comunicación }\end{array}$ & U. Católica de Murcia & - & - & $\mathrm{X}$ & - \\
\hline Telos & Fundación Telefónica & $\mathrm{X}$ & $\mathrm{XX}^{* *}$ & - & - \\
\hline Trama $\varepsilon$ fondo. Revista de cultura & $\begin{array}{l}\text { Asociación Cultural } \\
\text { Trama y Fondo }\end{array}$ & $\mathrm{X}$ & - & 一 & - \\
\hline Treballs de comunicació & $\begin{array}{l}\text { Societat Catalana de } \\
\text { Comunicació }\end{array}$ & - & - & $\mathrm{X}$ & - \\
\hline Tripodos & U. Ramon Llull & $\mathrm{X}$ & $\mathrm{X}^{* * *}$ & $\mathrm{X}$ & $\mathrm{X}$ \\
\hline $\begin{array}{l}\text { Zer. Revista de estudios de comuni- } \\
\quad \text { cación }\end{array}$ & U. del País Vasco & $\mathrm{X}$ & $\mathrm{X}$ & $\mathrm{X}$ & $\mathrm{X}$ \\
\hline
\end{tabular}

* En las secciones de Bellas Artes y de Cine.

** En la sección de Sociología.

**** En las secciones de Ciencias de la Información y de Sociología.

***** En la sección de Lingüística.

\section{Anexo II. Códigos de revistas}

AA

AJS

Ámbitos

Anàlisi

AoMJ

CdA

Chasqui

CIC
Área abierta.

The American Journal of Sociology.

Ámbitos. Revista internacional de comunicación.

Anàlisi: quaderns de comunicació $i$ cultura.

Academy of Management Journal.

Cuadernos de la Academia.

Chasqui. Revista latinoamericana de comunicación.

CIC. Cuadernos de información y comunicación. 


\begin{tabular}{|c|c|}
\hline Comunicar & $\begin{array}{l}\text { Comunicar. Revista científica iberoamericana de comunica- } \\
\text { ción y educación. }\end{array}$ \\
\hline CR & Communication Research. \\
\hline CT & Communication Theory. \\
\hline CyS & Comunicación y sociedad. \\
\hline DdC & Diálogos de la comunicación. \\
\hline Doxa & Doxa comunicación. \\
\hline EJC & European Journal of Communication. \\
\hline EsCC & Estudios sobre las culturas contemporáneas. \\
\hline EsMP & Estudios sobre el mensaje periodistico. \\
\hline Gazette & International Communication Gazette. \\
\hline HBR & Harvard Business Review. \\
\hline HyCS & Historia y comunicación social. \\
\hline $\mathrm{I} / \mathrm{C}$ & I/C. Información y comunicación. \\
\hline J\&MCQ & Journalism \& Mass Communication Quarterly. \\
\hline JB\&EM & Journal of Broadcasting \& Electronic Media. \\
\hline JoAR & Journal of Advertising Research. \\
\hline JoC & Journal of Communication. \\
\hline JQ & Journalism Quarterly. \\
\hline JS & Journalism Studies. \\
\hline MC\&S & Media, Culture \& Society. \\
\hline Mediatika & Mediatika. Cuadernos de medios de comunicación. \\
\hline NM\&S & New Media \& Society. \\
\hline PC & Political Communication. \\
\hline POQ & Public Opinion Quarterly. \\
\hline PRR & Public Relations Review. \\
\hline QdCAC & Quaderns del CAC. \\
\hline QP & Questiones publicitarias. \\
\hline RLCS & Revista latina de comunicación social. \\
\hline RyP & Razón y palabra. \\
\hline SdP & Sala de prensa. \\
\hline SexRoles & Sex Roles: a Journal of Research. \\
\hline SP & Sphera Pública. \\
\hline Telos & Telos. Cuadernos de comunicación, tecnología y sociedad. \\
\hline Trípodos & Trípodos. \\
\hline Zer & Zer. Revista de estudios de comunicación. \\
\hline
\end{tabular}

Rev. Esp. Doc. Cient., 33, 4, octubre-diciembre, 553-581, 2010. ISSN: 0210-0614. doi:10.3989/redc.2010.4.756 579 


\section{Anexo III. Códigos institucionales}

AUC

AUSTRAL-ARG

CAA

CSIC

CUV

EHU

EMA-RTV

FAVA

GEPEFIC-BRA
Asociación de Usuarios de la Comunicación.

U. Austral (Argentina).

Consejo Audiovisual de Andalucía.

Consejo Superior de Investigaciones Científicas.

Centro Universitario Villanueva.

U. del País Vasco/Euskal Herriko Unibertsitatea.

Asociación de Emisoras Municipales de Andalucía de Radio y Televisión.

Fundación Audiovisual de Andalucía.

ITESM-MEX Instituto Tecnológico y de Estudios Superiores de Monterrey (México),

PUCSP-BRA Pontificia Universidade Católica de São Paulo (Brasil),

UA

U. d'Alacant.

$\mathrm{UAB}$

U. Autònoma de Barcelona.

UACH-CHI U. Austral de Chile (Chile).

UAL U. de Almería.

UB U. de Barcelona.

UBA

U. de Buenos Aires (Argentina).

$\mathrm{UC3M}$

U. Carlos III de Madrid.

UCAM

U. Católica de Murcia.

$\mathrm{UCH}$

U. Cardenal Herrera-CEU.

UCM

U. Complutense de Madrid.

UDEA-COL

U. de Antioquia (Colombia).

UDEC-CHI

U. de Concepción (Chile).

UDEP-PER

U. de Piura (Perú).

UDG

U. de Girona.

UDG-MEX

U. de Guadalajara (México).

UGR

U. de Granada.

UHU

U. de Huelva.

UJI

U. Jaume I.

ULL

U. de La Laguna.

UM

U. de Murcia.

UMA

U. de Málaga.

$\mathrm{UMH}$
U. Miguel Hernández. 


$\begin{array}{ll}\text { UNAV } & \text { U. de Navarra. } \\ \text { UNAVARRA } & \text { U. Pública de Navarra. } \\ \text { UNED } & \text { U. Nacional de Educación a Distancia. } \\ \text { UNEX } & \text { U. de Extremadura. } \\ \text { UNICAMP-BRA } & \text { U. Estadual de Campinas (Brasil). } \\ \text { UNIOVI } & \text { U. de Oviedo. } \\ \text { UNIZAR } & \text { U. de Zaragoza. } \\ \text { UOC } & \text { U. Oberta de Catalunya. } \\ \text { UPB-COL } & \text { U. Pontificia Bolivariana (Colombia). } \\ \text { UPF } & \text { U. Pompeu Fabra. } \\ \text { UPO } & \text { U. Pablo de Olavide. } \\ \text { UPSA } & \text { U. Pontificia de Salamanca. } \\ \text { UPV } & \text { U. Politécnica de Valencia. } \\ \text { URJC } & \text { U. Rey Juan Carlos. } \\ \text { URL } & \text { U. Ramon Llull. } \\ \text { URV } & \text { U. Rovira i Virgili. } \\ \text { US } & \text { U. de Sevilla. } \\ \text { USAL } & \text { U. de Salamanca. } \\ \text { USC } & \text { U. de Santiago de Compostela. } \\ \text { USJ } & \text { U. San Jorge. } \\ \text { USMP-PER } & \text { U. San Martín de Porres (Perú). } \\ \text { USP-BRA } & \text { U. de São Paulo (Brasil). } \\ \text { USPCEU } & \text { U. San Pablo-CEU. } \\ \text { UV } & \text { U. de València. } \\ \text { UVA } & \text { U. de Valladolid. } \\ \text { UVIC } & \text { U. de Vic. } \\ \text { UVIGO } & \text { U. de Vigo. } \\ & \end{array}$

